\title{
Le charisme messianique d'Emmanuel dans Malevil (1972) de Robert Merle
}

\author{
Anne WATTEL, Université de Lille III, France
}

«Une situation est charismatique quand l'ordre ancien est remis en cause » (Dorna), c'est l'Evénement qui permet l'avènement : pour qu'une ère nouvelle émerge, portée par un être d'exception, il faut que quelque chose de l'avant ait pris fin, qu'un nouveau curseur marque le passage entre l'hier et le demain, que soit établi un temps « zéro », un «Jour $\mathbf{J}$ », un «jour de l'événement ». Il faut qu'il y ait eu cassure. Et que cette cassure, cette brèche, porte en elle les germes d'une possible disparition qui nécessite d'aller de l'avant, au risque de se perdre, de tout perdre. C'est dans un tel contexte que peut émerger la figure du grand homme, figure messianique qui ne s'impose que quand il faut ouvrir une voie, montrer la voie et donner de la voix pour susciter la cohésion du groupe en vue d'une mission collective, civilisatrice, voire de la survie du groupe, de l'humanité. Le «messie », c'est celui qui, par son charisme même, son pouvoir de fascination transformera l'inertie, le temps mort de l'après-événement, en possible, la désintégration en processus. C'est lui qui entraîne le groupe vers une issue, le désenglue pour que puisse émerger un demain, une ère nouvelle.

Le roman post-apocalyptique choisit d'explorer les possibles (et les impossibles) de l'après-événement. Il place les êtres, groupes de survivants, dans un univers où la linéarité a été rompue, où la «marche en avant des siècles s'est interrompue » (10). C'est le cas dans Malevil de Robert Merle. En véritable éthologue humain, l'auteur opte pour une démarche expérimentale face à un problème posé, «Qu'adviendra-t-il si... demain redevient avanthier ?»(Dubois). Il fait émerger une microsociété, une tribu de survivants dans un monde post-apocalyptique. Le monde d'avant, dévasté par une «bombe propre», a disparu. Désormais il n'y a plus rien, j'aurais dû dire, à la manière d'Emmanuel le narrateur, plus personne. Hormis, du moins au seuil de l'œuvre, un groupe, ceux de Malevil, protégés par la cave et l'enceinte d'un vieux château forteresse. Malevil devient alors le laboratoire au sein duquel Merle manipulera les variables, formulera ses hypothèses, démontrera.

Ce groupe secrétera le grand homme dont il a besoin car toutes les conditions sont ici réunies après l'événement pour l'avènement d'une figure charismatique : remise en question de l'ordre ancien, éducation de l'élu par un prédécesseur, mise en scène d'une figure du père, du père spirituel et culte du sauveur. Si le personnage d'Emmanuel exerce une influence presque religieuse sur ses compagnons, c'est qu'il est aussi une figure de la reliance (Bolle De Bal, 99-131) pour laquelle « pas d'unité, pas de survie» (624). Figure civilisatrice, il permet 
le dépassement du moi et apprend au groupe à faire corps, un seul corps qui sacrifie l'intérêt individuel au bien commun. Cette cohésion n'exclut ni les sacrifices ni, de la part d'un messie très politique, une certaine dose de machiavélisme « nécessaire à quiconque entend diriger ses semblables même s'il les aime» (621).

\section{Après l'Evénement, l'avènement d'une figure messianique}

Au seuil de Malevil, l'événement anéantit le monde d'avant. Passé le choc, la douleur, la tentation du suicide, l'événement devient avènement, il faut refonder, reconstruire et redonner le goût de l'à venir aux survivants. La situation est donc bien une situation de déséquilibre favorisant l'émergence d'un mouvement messianique, une situation où les repères traditionnels sont désormais dissous, où les liens d'hier n'ont plus lieu d'être car quelque chose dans le liant a cédé, quelque chose qu'il faudra restaurer pour que la vie communautaire, condition de la survie de l'espèce, reste possible. Les survivants sont désormais du côté de la privation: privation affective par la perte des proches particulièrement criante pour le grand couillon de Peyssou qui «épanchait un peu trop son chagrin familial » (146) ; privation-frustration à cause « de toutes les commodités qu'on avait plus » (145) et qui font grommeler la vieille Menou qui parvient difficilement à se sevrer des commutateurs qui ne répondent plus, du moulin à café, de la machine à laver, etc.; confiscation aussi, terrible, des certitudes, du confort qu'offrait le «monde d'avant - si protégé, si facile, si enfantin » (70) et d'un certain rapport au temps, à l'histoire, au passé et donc à soi-même car «il y a eu cassure » (10).

C'est dans ce contexte qu'émerge la figure charismatique qui, dans le temps d'avant, était déjà marquée du sceau de l'élection, mais n'avait pas pris toute son ampleur. En effet, le narrateur Emmanuel Comte est un élu. Le parcours et les enfances du héros sont pris en charge par un prédécesseur au sens où l'entend Florence Lojacono, celui-là même qui facilitera le passage au Nouvel Homme. Ce prédécesseur, c'est l'oncle d'Emmanuel, l'oncle Samuel, que le narrateur choisit pour père. Marginalisé dans sa propre famille, Emmanuel est une figure d'exclu; il est le «fils sérieux » de la parabole de l'enfant prodigue, celui pour lequel on ne tue pas le veau gras (14). Père, mère et sœurs sont autres, tous du côté du mou, du ressassement et du geignard, de l'esprit de routine : c'est Paulette et Pélagie, les deux sœurs, deux «molasses, blondasses » qui «bourdonnent comme des guêpes dans un bocal » (33), c'est la mère «geignarde et prêchi-prêchante » (11) hostile au défrichage proposé par le fils de 12 ans, et qui a réduit la père «au silence [...] par la simple vertu de son flux verbal » (12). Tous sont englués dans un temps à l'arrêt au sein duquel les fautes enfantines, les 
«crimes » ne sont «ni oubliés, ni pardonnés » (12). Emmanuel, enfant «reçu premier du canton au certificat d'étude »(11), se sent, se sait différent. Rejeté, il prend la décision de s'extraire du cercle familial pour créer son propre Cercle, sa société secrète, cellule de sept amis triés sur le volet, une «vraie famille ». Et il se choisit un père, un alter ego : Emmanuel devient fils de Samuel, l'oncle, tout heureux «de s'emporter un fils chez lui aux Sept Fayards » (20). Les deux prénoms d'ailleurs se font écho. Le rejeton devenant un autre Samuel, une expansion du premier.

Les deux personnages d'ailleurs sont des doubles, le même, à s'y méprendre. $\mathrm{Au}$ physique, bien sûr : Emmanuel a «hérité de l'oncle ses yeux bleus » (11), il est son «portrait» comme le lui reproche sa propre mère. La ressemblance est si frappante que, même dans l'après-événement, la vieille et coriace Menou en a les larmes aux yeux, qui confond Emmanuel avec l'oncle décédé (323). Mais la filiation va plus loin encore : contre le mou, le geignard, en choisissant l'oncle comme père, Emmanuel choisit la voix qui « tonne » (12), le «parler bref»(19), «l'esprit d'entreprise»(18), la vie, le rire de celui qui est profondément tourné vers l'avenir.

Samuel est lui aussi doté d'un certain charisme, c'est le « dieu » de la Menou (20), qui va participer de l'élection de son successeur mais lui sera inférieur en « gloire ». Il est en effet un véritable médiateur des désirs d'Emmanuel. Ils partagent le même goût pour les femmes, Emmanuel allant même jusqu'à «chasser sur les terres de l'oncle » lorsque, ignorant cette liaison, il tentera de faire des avances à Birgitta. Et c'est l'oncle qui fait émerger le désir de Malevil, la forteresse, l'oncle qui fournit à Emmanuel l'argent pour, après sa mort, réaliser le rêve qu'il n'a pu mener à terme, acheter Malevil.

Samuel, comme dans la Bible, est bien le faiseur de roi, celui-là même qui permettra l'émergence de l'Immanu-El, «Dieu avec nous », dont la naissance est annoncée dans le second livre d'Isaïe, le « livre de l'Emmanuel» : «La souveraineté est sur ses épaules. / On proclame son nom : / Merveilleux - Conseiller, Dieu-fort, / Père à jamais, Prince de la paix » (Isaïe, 9, 5).

Le nom est signe, comme est signe ce coup de «la providence» qui permet à Emmanuel de n'être pas tué avec ses parents et l'oncle dans l'accident d'auto car il aurait dû lui aussi «se trouver dans la $4 \mathrm{~L}$ »(32). Il a donc été sauvé, s'étonnant de cette élection : «Oui, mais pourquoi moi ? (33). Font signes aussi le désir du gamin Emmanuel de parler à Dieu, son «audace d'avoir pensé [...] à établir des liaisons directes avec Dieu » (29) ou encore la présence du corbeau, tel celui de la Genèse $(8,7)$ qui va jusqu'à se jucher sur son épaule après la catastrophe. Façonné par Samuel, faiseur de roi, Emmanuel fait donc figure 
d'élu et a tout du «type réincarné de la promesse d'Isaïe » comme l'indique Claude-Gilbert Dubois (64).

Dans l'œuvre, il est la figure refondatrice du sauveur. Les bornes analeptiques qui ponctuent le texte à ses débuts et le parsèment d'autant d'historiettes du monde d'avant, le lient toutes à Malevil, forteresse garante de la survie de l'humanité. C'est parce que Samuel suscite chez lui le désir de Malevil, parce qu'Emmanuel, véritable bâtisseur, colonise le lieu, ce lieu «refuge aux hommes»(49), ce lieu asile, c'est parce qu'il devait advenir qu'Emmanuel possède Malevil, parce qu'il faut procéder au «rituel sacré », à la «fête antique »(76) qui consiste à tirer le vin ce jour-là, dans la cave, parce que Peyssou, Meyssonnier, Colin, les anciens du Cercle, viennent trouver Emmanuel pour lui demander de proposer sa candidature de maire à la municipalité de Malejac, que la survie du petit groupe fut possible, au sein de Malevil, véritable arche de Noé.

Mais survivre n'est pas tout. Emmanuel devient véritablement une figure messianique quand il prend en charge la refondation, la re-naissance. Il est celui dont l' " amour fanatique pour les hommes et l'attachement à la continuation de l'espèce » (629) permettent la transformation de l'événement en avènement. Il devient le père refondateur. Il était déjà, enfant, le père du Cercle offrant à ses camarades un premier asile dans un Malevil en ruines, grâce à sa « direction inspirée » (16), il sera ensuite celui qui ouvre un avenir sur les cendres : «c'est moi qui, pour l'avoir conçue le premier, ai suscité chez eux cette espérance » (171).

Comme l'oncle avant lui, il incarne une figure paternelle. Jacquet, le fils troglodyte, fils du Wahrwoorde, sorte d'homme de Cro-Magnon, violent, incestueux, tout puissant qui, avec sa tribu, vit en marge, finit par le «piéger dans des rapports quasi filiaux » (274) après qu'Emmanuel a tué le père. Momo, vieil enfant attardé de la Menou, qui occupe le «dernier échelon de l'habileté linguistique »(238) éprouve les mêmes sentiments filiaux car «en héritant des biens de l'oncle, [Emmanuel a] hérité aussi de la puissance paternelle que l'oncle exerçait sur lui » (290).

Le père de la communauté de rescapés est celui qui répartit les tâches, impose le travail «pour nous tenir occupés » (150), travail d'équipe dont la division assure le ferment de la vie communautaire. C'est ce père qui stimule l'esprit de compétition entre les compagnons sur des activités exutoires et qui pourront s'avérer utiles pour la défense tel le tir à l'arc ; c'est lui qui encourage les initiatives, c'est aussi lui le meneur. «A moi de mener» précise-t-il à Thomas lors de l'assaut contre les Wahrwoode (190) et ce sera lui le stratège qui sera élu chef militaire de Malevil. 
Emmanuel, ce sera aussi le père spirituel, le pasteur qui veille sur ses brebis et le passeur qui fera que, par-delà la brèche, la faille et la cassure de l'événement, s'ouvre une voie nouvelle. Il instaure à la veillée la lecture de la Bible, «un magnifique poème » qui « chantait la création du monde » (159), et crée ainsi un pont entre la Genèse, la vie des «tribus primitives dans la Bible » et celle des survivants, afin que ces derniers y puisent « de la force dans l'opiniâtreté à vivre que les juifs avaient montrée »(159). Fréquemment d'ailleurs dans le texte, on le verra procéder à cette assimilation : pour lui, la messe raconte «l'angoisse d'il y a 2000 ans, la même que nous sommes en train de vivre » (308), et « comme au temps de la Bible, nous pensons en termes de nourriture, de terre, de troupeau et de conservation de la tribu » (249). Il deviendra son propre curé, élu abbé de Malevil, abbé bien peu orthodoxe comme il le dit lui-même, mais qui, dans la prière voit le besoin d'optimisme des hommes «entourés d'inconnu » (624) dans un monde devenu hostile et dans la religion l'âme de l'embryon de civilisation qu'ils tentent de remettre en place, une âme « qui correspond à [leur] niveau actuel de civilisation » (339).

Emmanuel exercera une véritable fascination sur ses compagnons. Le respect quasi religieux qu'il suscite apparait déjà chez Jacquet qui lui baise la main, et semble le considérer comme « infaillible et omniscient» (221). Evelyn, gamine asthmatique ramenée de La Roque, l'observe avec une «ferveur muette » (360). Même la pragmatique et ronchonneuse Menou voit en lui un «patron d'essence divine» (239) et, après sa mort, celle qui aura affronté de son maigre squelette tous les aléas de la vie, sombrera dans la prostration, puis mourra, amputée qu'elle sera de son énergie, énergie qui avait « besoin de s'appuyer sur une force qui la sécurisât [...] cette force, c'était Emmanuel » (629). Quant à Miette, ramenée de chez le Wahrwoode et qui n'a d'yeux que pour lui, telle Marie-Madeleine, on l'imagine « répandant des parfums coûteux sur les pieds d'Emmanuel et les essuyant ensuite avec ses longs cheveux »(258).

Dans une des scènes finales, alors qu'il affronte Fulbert, faux prophète qui a imposé au village voisin une véritable tyrannie théocratique, Emmanuel, dans la chapelle du château de La Roque, contribuera d'ailleurs à renforcer ce caractère religieux conféré à sa personne : usant des ressources du vitrail derrière lui, porté par la lumière et la chaleur du soleil, il emplit le lieu de sa personne et de sa voix : «Je me redresse. Pour mieux dire, je me campe. Je baigne avec volupté dans la lumière du vitrail. Je sens mes yeux qui s'ouvrent plus grands et mon être s'épanouir à cette clarté subite. [...] Je donne à ma voix son plein volume, je ne crains pas d'en emplir la chapelle » (593). 
Expansion, gonflement, ouverture et épanouissement, Emmanuel devient ici l'Illuminé, plein de la lumière que Dieu (ou le soleil) répand dans son âme. Le héroslibérateur, celui-là même qui permettra au village de La Roque de se défaire du joug de Fulbert, fera l'objet d'un véritable culte post-mortem comme nous l'apprenons dans la dernière «Note de Thomas », son successeur : «Tous les La Roquais vinrent assister à l'enterrement d'Emmanuel [...]. Ce fut là le premier en date des pèlerinages annuels de La Roque sur la tombe de son libérateur » (628).

\section{Emmanuel figure de la reliance : «Pas d'unité, pas de survie ! »}

Lorsqu'il revient à Malevil, triomphant, au chapitre VIII, ramenant avec lui deux des conditions de survie de l'espèce, la jeune Miette, Eve muette, «bouchée en haut » comme dirait Claude Lévi-Strauss, mais pas en bas, et qu'on ne conçoit que féconde, et... une vache, il fait figure de héros. Il a tout du maître, du seigneur féodal victorieux qui vient d'éliminer le rival et rapporte le butin. Pourtant, Emmanuel n'aura de cesse de refuser cette seigneurisation. On le voit dès le lendemain de l'événement, affamé, se contrôler pour ne pas se conduire « en maître en touchant à [ses] jambons » (119). Vainqueur du Wahrwoode, il se voit offrir par la Falvine, grand-mère imposante et gélatineuse, le «droit seigneurial » de mettre la main sur Miette (218), véritable droit de cuissage qu'il refuse. Si Emmanuel ne se conçoit pas seigneur, c'est que dans la nouvelle ère post-apocalyptique, où la priorité est la survie d'une communauté, la propriété privée n'a plus lieu d'être ; c'est qu'il œuvre désormais pour le bien commun. A maintes reprises, il expose ce qui pour lui est le fondement de sa conception : «Malevil est à nous tous » (134) ; «Malevil appartient à ceux qui y travaillent, c'est tout » (143) ; «le mien et le tien me paraissent des notions bien dangereuses » (277). Il n’y a plus de place pour le je dans ce monde-là, la condition de la survie réside dans le nous. Et, sous l'impulsion d'Emmanuel, Malevil devient dès lors bien plus qu'un nom de lieu pour guide touristique : «Malevil, aujourd'hui, c'est bien autre chose. C'est une tribu - avec ses terres, des troupeaux, des réserves de foin et de grain, des compagnons unis comme les doigts de la main, et des femmes qui nous porteront des enfants. C'est aussi notre repaire, notre tanière, notre nid d'aigle » (400).

C'est un corps, un grand corps aux membres soudés au sein duquel « les uns font les autres » (511). Chacun, lors de la préparation de la grande offensive contre les troupes armées du capitaine Vilmain qui œuvre pour Fulbert, invente, cherche, propose des initiatives car tout Malevil est «tendu vers un but unique » et donne l'impression à Emmanuel « de faire partie d'un tout que je commande mais auquel je suis en même temps subordonné, dont je ne suis 
moi-même qu'un rouage » (532). Malevil est à ce point un seul corps pour Emmanuel qu'il se sent amputé dès lors qu'il s'en éloigne, se comparant à un «bernard-1'hermite sans coquille » (377), à un «vêtement vide qui flotte » (539), lorsqu'il se trouve séparé de ses compagnons. Le groupe devient besoin, le groupe absorbe l'individu au point qu'Emmanuel se désincarne peu à peu, se « rétréci[t] tous les jours » (171), se «fond en lui »(315).

Ce corps, ce bien commun, suppose évidemment des sacrifices. L'individu n'a plus guère de place ici. «Je ne suis plus à moi » confiera Emmanuel à Agnès, qu'il aurait pu épouser il y a quinze ans, qui voudrait y remédier aujourd'hui et à laquelle il propose de venir habiter à Malevil non pas avec lui, mais avec tous, avec Colin, avec les autres et pour les autres. Emmanuel renonce au bonheur individuel. Dans la même logique, il demandera à Meyssonnier le communiste, son ami de toujours, qu'il n'a jamais quitté, un sacrifice « énorme » et qui lui fait « un peu honte»: quitter Malevil, abandonner les siens pour devenir maire de La Roque, parce que «c'est de l'intérêt de tous » (604).

C'est dans la même optique qu'il développe devant ses camarades sa conception de la relation à la femme. La femme ne peut devenir la propriété exclusive d'un seul homme. Dans une communauté où il n'y a au départ qu'une femme fertile, Miette, pour six hommes, les liens d'exclusivité n'ont pas leur place. Défenseur de la polyandrie, il sera mis en minorité par les autres hommes du groupe. Or cette question à elle seule est susceptible de faire voler en éclats la belle unité du groupe, en introduisant l'envie, la jalousie. Miette cependant, la généreuse, la fonctionnelle, se donne à tous, à chacun, comme un cadeau, un par soir, suivant l'ordre des places que les hommes occupent à table. Et lorsque d'autres femmes, celles de La Roque, viendront à Malevil, elles se donneront aussi, à leur façon. Il a fallu choisir : «la cellule familiale ou une communauté non possessive » (615). C'est cette seconde option qui s'est imposée malgré les vieux réflexes, malgré la «morale conventionnelle pas morte » (246), les tentations du mariage auxquelles Thomas cèdera en épousant Cathie, sœur de Miette, avant d'accepter qu'elle soit à tous ou tout au moins à qui elle veut. Malevil est un seul corps avons-nous dit plus tôt, et dans celui-ci, la jalousie disparaîtra de fait, car «tout se passe à l'intérieur du groupe, au vu et au su de tous. Il n'y a ni tromperie, ni même dévergondage » (286). La cellule familiale est bien dépassée, au point qu'à la fin de l'œuvre, les enfants nés de l'une ou l'autre des femmes appellent indifféremment tous les hommes «papa » et les femmes «maman» (632).

Pour conforter l'unité au sein de Malevil, il faut, selon Emmanuel, que pouvoirs temporel et spirituel soient entre les mains d'un seul chef. L'individualisme, la division, dans un monde où seul le groupe permet d'assurer la survie, sont un crime, une erreur vitale. C'est 
ce qu'il reproche à Thomas, le thésard, qui ne comprend pas toujours tout: «Tu ne comprends rien, parce que tu ne penses qu'à toi » (395) et, plus loin, quelques temps avant sa mort, excédé : «On se fout de tes opinions personnelles ! Elles ne comptent absolument pas ! Ce qui compte, c'est Malevil et l'unité de Malevil !»(624).

Emmanuel est donc une figure civilisatrice au sens où il crée le liant, soude, refonde, fait percevoir aux autres une communauté d'intérêt et permet qu'émerge un avenir. C'est lui qui œuvre pour que le groupe soit vraiment UN, qui assure l'équilibre entre la pluralité d'individus, qui met en contact, assure l'intégration des uns et des autres. Soucieux d'autrui, il voit très vite les failles et les colmate. Ainsi veillera-t-il à l'intégration de Thomas, le froid, impassible Thomas, qui ne comprend pas le patois local, et dont la rigueur de matheux, la beauté de statue grecque, la rigidité même sont l'antithèse des paysans, des Peyssou et Menou. Il ira de l'un à l'autre recommander qu'on n'abuse pas du patois devant Thomas et, pour rééquilibrer la balance, s'occupera davantage de lui. Lorsque Peyssou, encore sous le choc de la perte des siens, soûlera de ses épanchements son compagnon de chambrée, il ira de même, écoutant l'un puis l'autre, régler le problème en répartissant autrement les occupants des chambres. Et toujours, il aura pour souci de «huiler les rouages » (167), rabrouant l'un s'il le faut, en retirant un autre de la circulation parfois et veillant toujours à l'intégration des nouveaux, les Jacquet et Falvine, car les passions nationalistes peuvent très vite se déchaîner : il y aurait ceux de Malevil et les autres. C'est ce que refuse aussi Emmanuel. L'étranger peut être intégré et le narrateur révèle ici une véritable confiance en l'homme, en sa perfectibilité. Un fils de Wahrwoorde, ne deviendra pas forcément un Wahrwoode et, à la différence de la Menou qui éclate en patois contre les étrangers qu'Emmanuel ramène, notamment contre la Falvine, « cette vieille truie qu'elle est si grosse qu'elle peut même pas bouger le cul [...] que tu dirais un pot de saindoux qu'on a vidé sur un plat! » (234), il n'a pas l'« esprit de caste » (233) et il imposera que les nouveaux soient traités comme les autres.

Dans le même registre, c'est lui qui œuvre pour que la question religieuse ne divise pas le groupe. Dès le départ, cette question est source de dissension. Les fervents athées tels Meyssonnier le communiste et Thomas le rationnel, s'opposent à certains choix de la communauté. Thomas voit d'un assez mauvais œil les lectures de la Bible, qui fourmille d'«erreurs scientifiques ». L'arrivée des troglodytes à Malevil renforce le clan des religieux et les athées convaincus deviennent minoritaires, mais c'est surtout avec la visite de Fulbert que les choses se précisent. Il y a désormais les fulbertistes et les anti-fulbertistes. Une fois encore, c'est Emmanuel qui s'échine à éviter le « schisme », il impose le silence à Thomas et Meyssonnier et se félicite de les avoir empêchés de «déclencher une querelle religieuse » 
(274). Et lorsqu'il se fera élire abbé de Malevil, il exigera un vote unanime, obligeant Meyssonnier et Thomas à voter pour, les accusant, le cas échéant, de "saper l'unité de Malevil ». Une fois encore, ce qui lui importe, c'est d'empêcher toute division, d'assurer le liant.

\section{Un Messie très... politique}

La figure messianique d'Emmanuel ne s'impose pas facilement : le mort pèse toujours sur le vif. Face aux athées qui n'ont pas encore compris la nécessité du fait religieux dans ce monde de l'après, face aux « orthodoxes » prisonniers des vieilleries réactionnaires, englués dans les certitudes d'antan et incapables de la moindre souplesse, il lui faut également déployer des talents de stratège, de nouveau Prince, de politicien. Et ce sont des stratégies rodées, un art consommé de la parole et de la tactique qui permettront à l'ancien instituteur devenu messie d'asseoir son charisme.

Un des principaux atouts d'Emmanuel dans les débats, discussions, c'est son aptitude à se fondre dans le groupe, à opter pour une posture de retrait, une posture d'auditeur et de voyeur, un voyeur au regard acéré : «J'écoutais et je les regardais » $(142,153)$, «Je regardai Meyssonnier et Colin », «Je regardai Peyssou » (143), «J'écoute mes compagnons », etc., ces phrases sont récurrentes. L'écoute permet de mesurer le rapport de force, de cerner les clans, de prendre de la hauteur ; le regard permet d'observer les compagnons, d'avoir une posture extérieure. Emmanuel se fait public de ses compagnons, juge leur prestation : «pas mal Thomas, sobre », «ici il va un peu loin » (276). Dans cette posture Emmanuel peut jauger aussi leurs arguments : «je lui en voulus d'avoir dit ça / en fus reconnaissant d'avoir dit ça » (143). Le Messie sait se faire discret.

Ecoute et regard sont systématiquement liés au silence d'Emmanuel. «Je me tais. » (275), «Je ne fais rien pour rompre le silence » $(277)$, «ne dis mot » (171), « ne dis plus un mot » (279). Il s'agit ici d'une stratégie rodée, «stratégie de l'attente intermittente » (172) qui consiste à laisser la conversation s'enliser et ne déboucher sur aucune solution, sinon la division des interlocuteurs, pour enfin intervenir : « Attendre que la conversation s'enlise pour proposer une réponse » (64).

L'habileté verbale est indissociable de la technique avec laquelle Emmanuel amène ses arguments : rien d'abrupt, l'idée, glissée, prend le temps de germer avant d'être mise en mots clairs. Ainsi en est-il du mot «cheval» glissé au détour de la conversation, mot qu'Emmanuel est «content d'avoir introduit» (279) lors de l'assemblée générale qui doit trancher les conditions du troc avec Fulbert. De la même manière le narrateur joue sur les 
nuances et choisit habilement son vocabulaire : on dit, aux paysans réticents qu'on s'apprête à amputer de leurs bêtes, «séparer» et non «donner», car le don suppose l'absence de contrepartie. Toujours pour ce même auditoire paysan, on s'assure de son soutien en lui rappelant habilement qu'on appartient au même groupe en usant du patois local, de dictons du terroir : «vous connaissez le dicton du pays : les uns font les autres. (Je le dis d'abord en patois)... » (511), «Il suffit d'une mauvaise pomme pour te pourrir tout un panier » (279). C'est un moyen de puiser dans le fonds commun de la communauté, de rappeler qu'il y a entre lui et son auditoire des regroupements, des valeurs partagées, des liens, ainsi lorsqu'il rappelle aux anciens du Cercle, lors du débat sur la monogamie, l'Adelaïde, à laquelle tous doivent leur passage à l'âge d'homme, avoue-t-il dans son journal : «je m'appuie sur la force des souvenirs » $(244)$.

Le rythme est capital aussi pour entraîner l'auditoire, ou le perdre. Emmanuel ménage ses pauses, «une deuxième pause » (281), détache certains mots pour leur donner plus d'épaisseur, pour mettre en valeur un argument de poids, «j'accentue l'argument du standing » (282) ou pour mesurer l'impact d'un bon mot, « je me tais pour lui laisser avaler ce bol de fiel » (355). Par ailleurs, il excelle dans l'art de distribuer la parole : «A toi, Colin. », «Meyssonnier? », «Peyssou ?», «Jacquet ?», «Thomas », (427). Il choisit ici l'ordre dans lequel il les interpelle, ordre qui contribue à reléguer Thomas à la fin pour lui «marquer de la distance », puisqu'il considère qu'il y a eu de sa part défaillance.

Le langage est affaire de ton et de corps aussi, affaire de mime, de jeu, un jeu théâtral que maîtrise parfaitement l'ancien instituteur qui «se penche en avant » et «accentue avec force » (282), varie le ton, hypocrite parfois, avec aisance. Il sait jouer le bien élevé s'il le faut, «avec politesse » (62), sait jouer le second couteau, «avec modestie », sait donner l'illusion «avec toutes les apparences de l'équité » (24), et jouer un rôle de composition si habile que même ses compagnons sont parfois «consternés de cette modestie »(280). L'objectif est de toucher l'auditoire, d'obtenir l'adhésion totale car le charisme implique un vote unanime. Il faut donc «faire mouche» (280) et pour cela, encore faut-il moucher ceux qui, s'opposant, risquent de dissoudre le groupe, l'unité et de lui faire perdre la face. Les vacheries bien senties, il s'en délecte : «je savoure cette petite vacherie » (243). Pour l'unité, il utilise parfois un double langage ; il y a ce qui se dit à tous et ce qui se dit à certains. En groupe déjà, des signes sont échangés en aparté : par deux fois, aux pages 62 et 66, il fait signe à Meyssonnier de ne pas parler ; plus tard, il empêche Thomas d'intervenir face à Fulbert en lui adressant un avertissement sous la table (268). Pour s'éviter des conflits et s'assurer l'unanimité, il pratique la stratégie de l'entretien privé : avec les deux athées, 
Meyssonnier et Thomas, sur la messe que Fulbert compte faire à Malevil (282), avec Colin et Peyssou, pour être élu abbé de Malevil, fréquemment on le verra prendre certains compagnons à part et s'adonner à des «entretiens urgents qui demandent un certain secret » (598).

Si effectivement la pratique des assemblées générales, des débats, des votes, les demandes d'aide et conseils à l'ensemble des compagnons semblent mettre l'accent sur la question démocratique, accent renforcé par les formules du type «si tout le monde est d'accord », (158), « à nous tous d'en décider » (278), « je me rangerai à l'avis général » (280), on voit clairement cependant qu'Emmanuel dispose d'atouts que les autres n'ont pas pour les mener là où il l'entend. Plus instruit que la majorité de ses compagnons, Thomas excepté, il maîtrise les finesses de la parole et piège facilement son auditoire. Rien d'impulsif chez Emmanuel, tout est calculé, dans l'intérêt de la communauté certes, mais calculé tout de même et il n'est pas sans parfois éprouver du remords. Mais ce qui prime c'est le groupe et pour la survie de ce dernier, il joue toujours ses meilleurs atouts et se présente « avec un jeu gagnant », comme lorsque, pour convaincre Meyssonnier de quitter Malevil, il lui offre la mairie et... la Marie. Son habileté passe pour du cynisme auprès de Thomas qui voit clair dans son jeu. S'il le trouve parfois machiavélique, il reconnaîtra cependant que cet aspect du chef était «nécessaire pour diriger ses semblables même s'il les aime » (621), que ce fut une chance d'avoir eu Emmanuel car « il a maintenu l'union et il nous a appris à nous défendre » (622).

Ni Wahrwoorde, qui vit comme un sauvage indifférent aux lois, foulant aux pieds les règles les plus élémentaires de la vie sociale, et mène son groupuscule familial par la terreur, ni Fulbert, le fourbe, véritable Janus aux deux visages, SS local, sournois, libidineux qui ne sut pas faire de La Roque une communauté et instaura une caste de nantis avec sa police, ses privilèges, ses délations, Emmanuel, porté par sa foi en l'homme, son optimisme volontariste est celui qui, par-delà la brèche, le fossé ouvert par l'événement, permet d'inaugurer une ère nouvelle. Une ère qui débute «ce jour de Pâques où l'histoire cesse faute d'objet » (75) et qui permet la résurrection par l'avènement d'Emmanuel qui unit étroitement la dimension politique et la dimension religieuse au point de faire émerger une nouvelle religion, comme le révèle Thomas, son successeur, à la fin du roman: «J'appris que les trois femmes se réunissaient dans la chambre de Miette et je les y surpris, en train de pleurer et de prier au pied d'une table sur laquelle trônait, comme sur un autel, le portrait d'Emmanuel. [...] Et c'est elles qui, contaminant les La Roquaises, finirent par organiser ce culte du héros mort qui est presque devenu chez nous une seconde religion » (634). 


\section{Ouvrages cités}

Dorna, A. « La question du chef charismatique : l'image épique et la dynamique émotionnelle ». Les cahiers psychologie politique 13 (juillet 2008).

Merle, Robert. Malevil. Paris : Folio, 2001.

Dubois, Claude-Gilbert. «Qu'adviendra-t-il si... demain redevient avant-hier ? Expérimentations de politique-fiction dans Malevil (1972) de Robert Merle ». Fictions d'anticipation politique 73. Dir. M. Pratt et A. Sebbah. Toulouse : PU de Toulouse, 2006.

Bolle De Bal, Marcel. «Reliance, déliance, liance: émergence de trois notions sociologiques ». Société $80: 2$ (2003). 99-131.

Lojacono, Florence. «Un invariant de la robinsonnade ontologique: la figure du prédécesseur ». (2009). 